\title{
Lung Biopsy in Coronavirus-19 Pneumonia: A Case Report and Review of Literature
}

\author{
Nadira Mamoon ${ }^{1}$, Imran Nazir Ahmad ${ }^{1}$, Atif Iqbal Rana² and Haseeb Haider Zia ${ }^{3}$ \\ ${ }^{1}$ Department of Histopathology, Shifa International Hospital, Islamabad, Pakistan \\ ${ }^{2}$ Department of Radiology, Shifa International Hospital, Islamabad, Pakistan \\ ${ }^{3}$ Department of Hepatobiliary Surgery, Shifa International Hospital, Islamabad, Pakistan
}

\begin{abstract}
Pneumonia is one of the major manifestations of infection by the novel coronavirus (COVID-19) virus. CT scans are used as first line investigation in suspected cases. Biopsies are almost never done. The gross and microscopic pathology has been studied mostly on autopsy specimens in fatal cases. We present a case of a mildly symptomatic adult male, who was a chronic liver disease patient awaiting liver transplant. His lung biopsy was done for diagnosis of a lesion, suspected to be a fungal infection. He was tested for COVID-19 and was PCR-negative at the time. His biopsy showed patchy changes of viral pneumonia. One week later, he tested positive for COVID-19 on PCR. He became asymptomatic soon and the ground glass lung opacities cleared up within another week without any treatment in spite of having a serious comorbid condition.
\end{abstract}

Key Words: Viral pneumonia, COVID-19, Lung biopsy.

How to cite this article: Mamoon N, Ahmad IN, Rana Al, Zia HH. Lung Biopsy in Coronavirus-19 Pneumonia: A Case Report and Review of Literature. J Coll Physicians Surg Pak 2021; 31(JCPSPCR):CR117-CR119.

\section{INTRODUCTION}

Coronavirus 2019 pandemic has taken the world by storm. The disease mortality is mostly due to the involvement of respiratory system, leading to failure. X-ray chest and CT scan are the mainstay of diagnosis with confirmation by positive PCR test on nasopharyngeal swabs. Biopsies are rarely done and autopsies are strongly discouraged, given the highly transmissible nature of the virus. ${ }^{1,2}$

We present a case of a COVID-19 infection in which lung biopsy was done for a suspicion of fungal infection or metastatic disease. Verbal consent was taken from the patient for submitting this case report. The aim of this case report is to describe the biopsy findings in correlation with radiology.

\section{CASE REPORT}

A46-year man with cirrhosis and decompensated alcoholic liver disease, who was a liver transplant candidate, was being worked up by the transplant surgeon. He was not on any steroids or otherimmunosuppressive therapy.

Correspondence to: Dr. Nadia Mamoon, Department of Histopathology, Shifa International Hospital, Islamabad, Pakistan

E-mail:nmamoon@live.com

Received: July 21, 2020; Revised: July 02, 2021;

Accepted: July 07, 2021

DOI: https://doi.org/10.29271/jcpsp.2021.JCPSPCR.CR117
There was history of encephalopathy (3-4 times), ascites, and pleural effusion. He underwent pleurodesis in another hospital some time back. He was an occasional smoker. CT chest showed a loculated mild to moderate right-sided pleural effusion with compressive atelectasis of adjacent lung on $12^{\text {th }}$ May, 2020. His baseline investigations including alanine aminotransferase (ALT), aspartate aminotransferase (AST), alkaline phosphatase (ALK Phos), total bilirubin, direct bilirubin, gamma glutamyl transferase (GGT), and creatinine were $37 \mathrm{U} / \mathrm{L}, 62 \mathrm{U} / \mathrm{L}, 186 \mathrm{U} / \mathrm{L}$, $4.35 \mathrm{mg} / \mathrm{dl}, 2.02 \mathrm{mg} / \mathrm{dl}, 46 \mathrm{U} / \mathrm{L}$, and $0.69 \mathrm{mg} / \mathrm{dl}$, respectively. Baseline hemoglobin was $10.2 \mathrm{~g} / \mathrm{dl}$, with a total white blood cell (WBC) count of $3,830 /$ ul. Hematocrit was $28.6 \%$. One week later, he complained of nausea, lethargy and shortness of breath. He was afebrile. CT was repeated on $18^{\text {th }}$ May; and on comparison with the previous $\mathrm{CT}$, he showed development of thick walled cavities in right lung at the site of previously seen loculated effusion with surrounding atelectasis. There was development of multiple right hilar and perihilar ground glass opacities (Figure 1). Blood culture and pleural fluid culture did not show any growth after 48 hours incubation. CT-guided core biopsy from the lung parenchyma forming the wall of one of the cavities was done on $21^{\text {st }}$ May, using a $20 \mathrm{G}$ needle and two cores were obtained. The provisional diagnosis was metastatic disease or infective process, likely fungal. COVID-19 PCR of nasopharyngeal swab was done 24 hours prior to both CT scans; and was found negative.

The biopsy showed lung tissue with areas of alveolar damage and type II pneumocyte hyperplasia. Occasional hyaline membranes were seen. Intra-alveolar inflammatory infiltrate 
comprised of mostly histiocytes admixed with few lymphocytes. Alveolar septae were also thickened with histiocytic infiltrate admixed with neutrophils and lymphocytes. One area showed fibroblastic proliferation infiltrated by neutrophils, lymphocytes and histiocytes (Figure 2). Focal infiltration of bronchial walls was also seen by the infiltrate but lumina did not show any mucin or infiltrate. No granulomas were present. Special stains were negative for acid fast bacilli (AFB) and fungus. Immunohistochemistry for cytomegalovirus (CMV) was negative. Few cells with possible viral inclusions were also seen (Figure 3). An occasional microthrombus was seen.

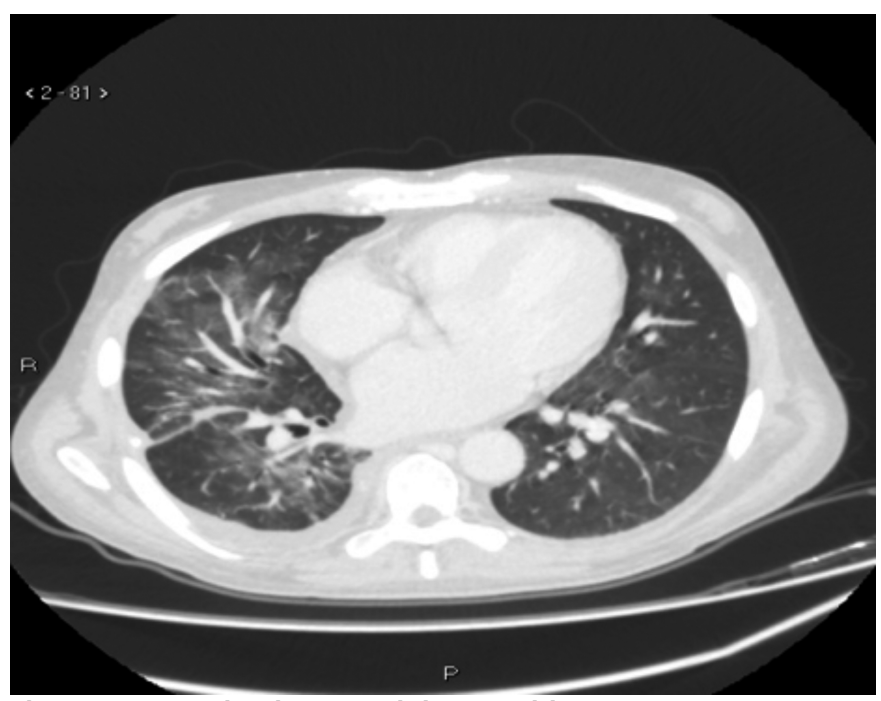

Figure 1: CT scan showing ground glass opacities.

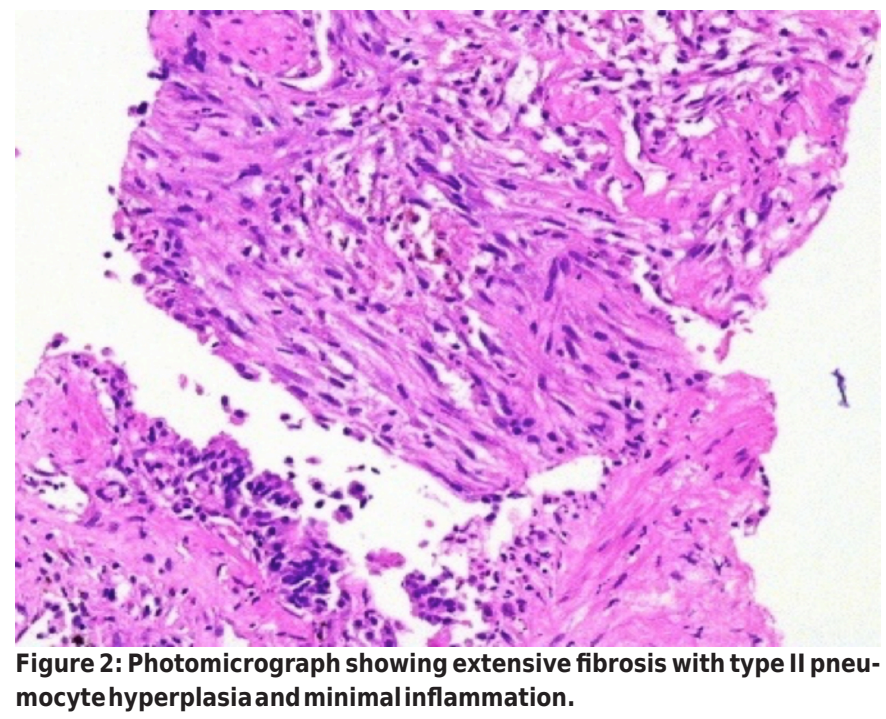

Post-biopsy, the patient was started on tab moxifloxacin, 400 $\mathrm{mg}$, once a day and IV meropenem, $1 \mathrm{~g}$, daily. PCR for COVID-19 was repeated seven days after taking the biopsy, and it was positive. Follow-up CTscan done after two weeks showed resolution of the ground glass changes. Serology for anti-SARS-CoV-2 antibodies done on $16^{\text {th }}$ June was positive. Patient went on to have a liver transplant two weeks thereafter, and had an uneventful recovery.

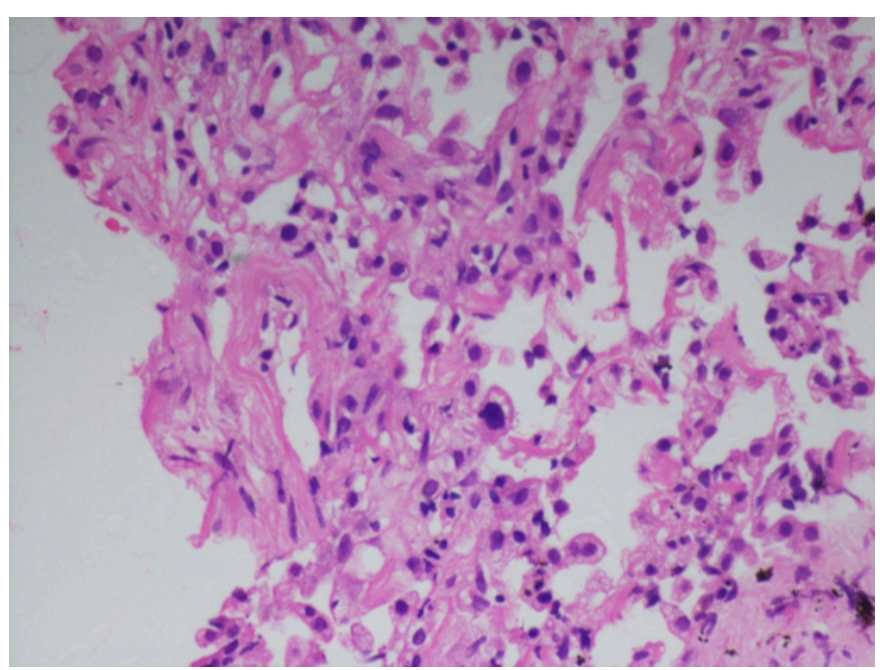

Figure 3. Photomicrograph showing viral inclusion in alveolar lining cells. (HE, ×400).

\section{DISCUSSION}

COVID-19 pandemic has taken the world, by storm. Medical experts of all fields are trying to learn about this novel pathogen. The present case demonstrates the findings of a core lung biopsy done in an unsuspected case. It is unique in the sense that core biopsies are usually not done and literature focuses mostly on autopsy findings, which mostly document advanced disease.

Radiological findings in COVID-19 infection are classically reported as ground glass opacities and consolidation. In this patient, the biopsy was obtained from the wall of a cavitary lesion. A few cases of cavitary lesions in COVID-19 infection are reported in literature although they are reported in resolving stage of infection. ${ }^{1}$ The radiological lesion in this case may be unrelated to the viral infection and may be a consequence of the previous interventions for pleural effusion.

The findings on microscopy were not specific in this case and were similar to what may be seen in any viral pneumonia. They included alveolar damage, hyaline membranes and septal and alveolar inflammatory infiltrates. Our findings are similar to those seen by other workers. Most of these findings have been documented in autopsies, which show severe and diffuse disease. ${ }^{2}$ One study of resected lung cancers showed early and mild changes discovered incidentally as seen in present case. The findings were similar with mild inflammatory infiltrate, congestion, patchy and severe pneumocyte hyperplasia and multinucleate giant cells. ${ }^{3}$ Subsequently, the disease progressed in these patients and one of them died of severe pneumonia. ${ }^{4}$ So, the findings in present case most likely represent a patient in whom, for some reason, the infection did not progress significantly.

Workers who have performed immunohistochemistry on lung tissue in autopsy cases using polyclonal antibodies against SARS-CoV-2 antigens have shown positivity in the cytoplasm of alveolar epithelial cells and multinucleate syncytial cells. ${ }^{5}$ Immunofluorescence has also been used for this purpose with antibodies against R3N3p protein of the SARS-CoV-2 virus. ${ }^{6}$ Unfortunately, we did not have specific immunohistochemistry 
marker available; however, the patient became seropositive for anti-SARS-CoV-2 a short while later, indirectly confirming the diagnosis.

It has been seen that COVID-19 infection is systemic and leads to liver damage due to injury to hepatocytes and bile duct cells. In patients with underlying chronic liver disease such as hepatitis B, and $C$ viruses, autoimmune hepatitis, and alcoholic liver disease, the effects of infection may be modified or aggravated by therapy such as immunosuppressives or steroids. ${ }^{7}$ This patient was not on any immunosuppressive therapy, and this may have contributed to his recovery from infection without any adverse effects.

This implies that we do not know exactly which risk factors determine whether a patient will suffer from mild or severe lung disease or systemic disease.

In conclusion, early changes of COVID-19 infection are similar to other viral pneumonias with patchy alveolar damage and focal hyaline membranes. Mildly symptomatic self-limiting disease in a chronic liver disease patient implies hitherto unknown factors at work which determine the severity and course of disease.

\section{PATIENT'S CONSENT:}

Verbal consent of the patient was obtained.

\section{CONFLICT OF INTEREST:}

The authors declared no conflict of interest.

\section{AUTHORS' CONTRIBUTION:}

NM: Conception and design, acquisition, and interpretation of data. INA: Design, data analysis, drafting, and revision.
AIR: Acquisition of data, analysis, drafting, and revision. HHZ: Concept, interpretation, drafting, and revision.

\section{REFERENCES}

1. Chen Y, Chen W, Zhou J, Sun C, Lei Y. Large pulmonary cavity in Covid 19 cured patient case report. Ann Palliat Med 2021; 10(5):5786-91. doi: 10.21037/apm-20-452.

2. Barton LM, Duval EJ, Stroburg E, Ghosh S, Mukhopadhyay S. Covid-19 Autopsies, Oklahoma USA. Am J Clin Pathol 2020; 153(6):725-33. doi: 10.1093/ajcp/aqaa062.

3. Tian ST, Hu W, Niu L, Liu H, Xu H, Xiao S. Pulmonary pathology of early-phase 2019 novel coronavirus (Covid-19) pneumonia in two patients with lung cancer. J Thoracic Oncol 2020; 15(5):700-4. doi: 10.1016/j.jtho. 2020.02.010.

4. Tian ST, Xiao S. Pathology of 2019 novel corona virus pneumonia: A dynamic disease process. J Thoracic Oncol 2020; 15(5):e67-8. doi: 10.1016/j.jtho.2020.03.015.

5. Adachi T, Chong J, Nakajima M, Sano M, Yamazaki J, Miyamoto I, et al. Clinicopathological and immunohistochemical findings from autopsy of patient with Covid 19 Japan. Emerg Infect Dis 2020; 26(9): 2157-161. doi: 10.3201/eid2609.201353.

6. Zhang $\mathrm{H}$, Zhou P, Wei $\mathrm{Y}$, Yue $\mathrm{H}$, Wang $\mathrm{Y}$, Hu M, et al. Histopathologic changes and SARS -Cov-2 staining in the lung of a patient with Covid 19. Ann Intern Med 2020; 172(9):629-32.

7. Wu J, Song S, Cao HC, Li LJ. Liver diseases in Covid 19: Etiology, treatment and prognosis. World J Gastroenterol 2020; 26(19):2286-93. doi: 10.3748/wjg.v26.i19.2286. 